\title{
Lengthscales of Mantle Volatile Heterogeneity
}

\section{S. MATTHEWS ${ }^{12 *}$, O. SHORTTLE ${ }^{23}$, J. MACLENNAN $^{2}$, J.F. RUDGE ${ }^{2}$, B. MURTON ${ }^{4}$}

${ }^{1}$ Earth and Plantary Sciences, Johns Hopkins University, Baltimore, Maryland, USA. (*correspondance:

simonmatthews@jhu.edu)

${ }^{2}$ Earth Sciences, University of Cambridge, Cambridge, UK.

${ }^{3}$ Institute of Astronomy, University of Cambridge,

Cambridge, UK.

${ }^{4}$ National Oceanography Centre, Southampton, UK.

It is well established that the mantle is chemically heterogeneous on lengthscales of 10 s of kilometres or less; a product of recycling melting residues and subducted lithosphere, followed by stirring and mixing during vigorous convective cycling. Whilst heterogeneity in the concentration and isotope ratios of volatile elements (e.g. C, H, He) within the mantle has been observed on scales of 100s of kilometres, and we might expect it to exist on the scale of $10 \mathrm{~s}$ of kilometres, to date there is little observational support for such fine scale volatile heterogeneity.

Since subaerially erupted lavas lose most of their volatile element budget prior to or during eruption, volatile studies are limited to submarine glasses and crystal-hosted melt inclusions. We present new volatile and trace element analyses for a suite of glasses from the Reykjanes Ridge, and for melt inclusions from four Icelandic eruptions. To estimate pre-eruptive volatile concentrations, we develop methods for filtering melt inclusion datasets for the effects of decrepitation, $\mathrm{H}^{+}$diffusion, and concurrent mixing and degassing. Using the submarine glasses and melt inclusions in tandem, we identify small-scale heterogeneity in mantle volatile-trace element ratios, from which we estimate volatile concentrations in Icelandic mantle components.

Comparing the magnitudes of small- and large-scale mantle volatile heterogeneity allows us to place new constraints on the relative importance of processes mediating the transport of volatiles throughout the Earth's mantle. 